\title{
Interkulturelle Kommunikation und christliche Mission in der frühen Neuzeit
}

\author{
Michael Sievernich S.J.
}

In Dantes „Göttlicher Komödie“ erzählt der mythische Held Odysseus von seiner Seereise nach Westen, bei der er die Meerenge von Gibraltar durchquerte und auf der Suche nach unbekannten Welten hinaus aufs offene Meer segelte, ohne in seinem Entdeckungsdrang darauf zu achten, dass die ,Säulen des Herkules' (Gibraltar) zum Warnzeichen aufgerichtet waren, nicht weiterzufahren (più oltre non). ${ }^{1}$ Wegen dieser Grenzüberschreitung, die zum Schiffbruch führen musste, schickte Dante ihn in den achten Kreis der Hölle, dorthin, wo die bösen Ratgeber für ihre Taten büßen (Inferno, 26. Gesang).

Wofür Odysseus in die literarische Hölle musste, dafür wurde Kolumbus in den weltgeschichtlichen Himmel gehoben. Auf der Suche nach dem westlichen Seeweg nach Indien machte auch er sich mit seinen Schiffen aufs hohe Meer nach Westen auf, aber seine Grenzüberschreitung, die zur Entdeckung einer für Europa Neuen Welt führte, entsprach ganz der Devise Plus ultra (,Noch weiter hinaus'), die der spanische König und deutsche Kaiser Karl V. als heroischen Wahlspruch gewählt hatte.

Die so gegensätzliche Bewertung des Aufbruchs nach Westen als sündhafte Hybris oder als providentielle Tat erklärt sich aus dem Epochenbruch, d.h. dem Wechsel vom mittelalterlichen zum frühneuzeitlichen Weltbild, in dem Forscherdrang und Entdeckerlust nicht mehr der seit Augustinus sündhaften $c u-$ riositas zugerechnet werden, sondern als Ausdruck der grenzenlosen Freiheit des Menschen zur Weltbemächtigung gelten, wie Giovanni Pico della Mirandolas Programmschrift zeigt. ${ }^{2}$ Die Begegnung mit anderen Völkern, Kulturen und Religionen in der Neuen Welt, vor allem aber die Ambivalenz des Ausgriffs in der martialischen oder missionarischen Variante forderten dazu heraus, die Muster der Wahrnehmung, Deutung und Gestaltung normativ zu reflektieren.

Die conquista der Neuen Welt, die Zerstörung blühender altamerikanischer Reiche und die Schaffung von Kolonialreichen, das Eintreiben von Tributen und die Versklavung von Indianern und Afrikanern, all diese und andere Phänomene führten zu neuen Diskursen um Krieg und Frieden, um die Legi-

1 Opere poetiche di Dante. Dantes Poetische Werke. Neu übertragen und mit Originaltext versehen von Richard Zoozmann, Bd. 1, Freiburg 1908, 225 (Inferno XXVI, 109).

2 Giovanni Pico della Mirandola: De hominis dignitate. Über die Würde des Menschen. Übers. von N. Baumgarten, hrsg. von A. Buck, Hamburg 1990 (PhB 427). 
timität spanischer Herrschaft, um angemessene Methoden der Mission. In diesem Feld war besonders die Einrichtung der encomienda umstritten, eines Systems, welches das Recht auf Nutznießung erzwungener Arbeit mit der Missionspflicht der zur Arbeit Gezwungenen verband. Zudem ist zu berücksichtigen, dass die iberischen Könige aufgrund päpstlicher Konzessionen das Patronat (patronato, padroado) $)^{3}$ über die Kirche innehatten, das ihnen weitgehende Rechte verlieh (Ernennung von Bischöfen, Auswahl von Missionaren, Einrichtung von kirchlichen Jurisdiktionsbezirken, Einziehung des Kirchenzehnten) und Pflichten auferlegte (Einrichtung und Finanzierung kirchlicher Strukturen und des gesamten Missionswesens). Die Missionierung war „ein zentrales Instrument zur Integration der Indianer in eine spanisch geprägte Herrschafts- und Gesellschaftsordnung. ${ }^{“ 4}$

Aus der Vielzahl der verfügbaren Quellen zu diesen Diskursen über die juridischen, ethischen und theologischen Grundlagen der conquista seien im folgenden zwei Positionen vorgestellt, die im machtpolitischen Rahmen der Expansion und im religionspolitischen der Mission die Andersheit der fremden Kulturen und Religionen für höchst unterschiedliche Umgangsweisen plädierten. Hervorragende Protagonisten im frühneuzeitlichen Diskurs sind zwei fast gleichaltrige spanische Zeitgenossen, der Humanist Juan Ginés de Sepúlveda (1490-1573) und der etwas ältere Scholastiker Francisco de Vitoria (14831546). Beide bedienen sich im Zusammenhang der Eroberung und Christianisierung der indianischen Völker der Rechtsfigur des bellum iustum: der eine, um die ,gerechten Gründe' für einen solchen auszuweiten und so der Mission den Weg zu ebnen, der andere, um die ,gerechten Gründe' einzudämmen und die Mission auf diese Weise zu befördern. Beide haben die Neue Welt nicht mit eigenen Augen wahrgenommen, sondern mussten sich auf die Berichte anderer verlassen.

\section{Krieg und Mission bei Juan Ginés de Sepúlveda}

Der Humanist Juan Ginés de Sepúlveda $(1490-1573)^{5}$ studierte in Córdoba und Alcalá de Henares, lernte am spanischen Kolleg in Bologna den italienischen Humanismus kennen und stand zunächst in Diensten der römischen

3 Pedro de Leturia: Relaciones entre la Santa Sede e Hispanoamérica, Bd. 1: Epoca del real Patronato, Rom Caracas 1959.

4 Horst Pietschmann: Die staatliche Organisation des kolonialen Iberoamerika, Stuttgart 1980, 32 .

5 Cf. Horst Pietschmann: Aristotelischer Humanismus und Inhumanität? Sepúlveda und die amerikanischen Ureinwohner, in: Humanismus und Neue Welt (Mitteilung XV der Kommission für Humanismusforschung), hg. von WolfGang Reinhard, Weinheim 1987, 143-166. 
Kurie, bevor er 1535 in die Dienste der spanischen Krone trat. Am Hof Karl V. befasste er sich neben seinen Tätigkeiten als Chronist und Prinzenerzieher auch mit politischen und staatsrechtlichen Fragen seiner Zeit. Daraus gingen im Stil der Zeit unter anderem zwei humanistische Dialoge über die Fragen von Krieg und Frieden hervor: Der mehr allgemeine Dialog „Democrates primus" (1535) und der mehr auf die Neue Welt bezogene Dialog „Democrates secundus de iustis belli causis“ (1541), auch „Democrates alter" genannt. Die Veröffentlichung der letzteren Schrift scheiterte allerdings an der Zensur, weil Melchor Cano und Bartolomé Miranda de Carranza, beide Schüler Vitorias, keine Druckempfehlung aussprachen.

Sepúlveda vertrat in dieser Kolonialdebatte die Auffassung, dass der Krieg gegen die Indianer nicht nur erlaubt, sondern sogar geboten sei. Mit dieser Position stand Sepúlveda keineswegs allein da, vielmehr repräsentierte er eine breite Grundströmung der Zeit, die vor allem von den Franziskanern geteilt wurde ${ }^{6}$ während die Dominikaner, allen voran sein Hauptgegner Bartolomé de Las Casas, für die Gegenposition standen. Sepúlveda argumentierte für die Erlaubtheit des Krieges in Amerika im Rahmen der scholastischen Theorie des bellum iustum, die bei Thomas von Aquin eine klassische Ausformung gefunden hatte. Dieser verwirft in seiner „Summa Theologiae“ (S th II-II q. 40) den Krieg als Verletzung des Liebesgebots, lässt aber unter bestimmten Bedingungen Ausnahmen zu, d.h. unter den drei Bedingungen der legitimen Autorität eines Fürsten (auctoritas legitima), des gerechten Grundes (causa iusta) und der rechten Absicht (recta intentio).

Angesichts der neuen Situation in der Neuen Welt nimmt Sepúlveda bei dem Kriterium der causa iusta eine erhebliche Erweiterung vor. Waren aus der Tradition drei „gerechte Gründe“ für einen Krieg bekannt, d.h. die Verteidigung, die Rückgewinnung der Kriegsbeute und die Bestrafung der Schuldigen, so fügt Sepúlveda vier weitere Gründe hinzu, nach denen die Indianer, falls sie sich nicht freiwillig fügen, auch mit kriegerischer Gewalt unterworfen werden können. Systematisch entfaltet er diese Gründe im Dialog „Democrates secun$d u s^{\prime \prime}$.

In diesem Dialog sind die beiden Gesprächspartner der lutherisch orientierte Leopoldo, der mit biblischer Begründung den Krieg ablehnt und nur in Ausnahmefällen rechtfertigende Gründe (causae iustae) anerkennt, und Democrates, Sepúlvedas Sprachrohr, der die weiteren Gründe vorbringt. Diese vier neuen

6 Cf. J. González Rodríguez: Los amigos franciscanos de Sepúlveda. In: Actas del II Congreso internacional sobre los franciscanos en el Nuevo Mundo (siglo XVI), Madrid 1988, 673-693.

7 Juan Ginés de Sepúlveda: Democrates segundo o de las justas causas de la guerra contra los indios, edición crítica bilingüe, traducción castellana, notas e índices por A. Losada, Madrid 1951; (im laufenden Text unter Sigel DS und Seitenangabe). 
Argumente zur Rechtfertigung des spanischen Vorgehens in der Neuen Welt (aliae iusti belli causae, DS 19) lassen sich folgendermaßen zusammenfassen:

(1) Sklaven von Natur aus. Das erste Argument bezieht sich auf die aristotelische Lehre der Sklaven „,von Natur aus“ (Politik 1254b) und wendet diese auf die als homunculi (DS 35) bezeichneten Indianer an, wie es schon der in Paris lehrende John Mair (Maior) in seinem Sentenzenkommentar (1510) getan hatte. Hier wird die Inferiorität ontologisiert und als Grund für das Recht der kulturell überlegenen Spanier eingeführt, Krieg zu führen.

(2) Strafe für Sünden. Das zweite Argument besteht in dem Recht der Fürsten, die spezifischen Sünden der Barbaren wider die Natur, d.h. Kannibalismus, Idolatrie und Menschenopfer, zu bestrafen und einzudämmen.

(3) Humanitäre Intervention. Das dritte Argument lautet auf die naturrechtlich begründete, unbedingte Pflicht, Unschuldige zu schützen, falls sie durch die Praxis von Kannibalismus und Menschenopfern bedroht sind.

(4) Heidentum als Hindernis. Das vierte Argument schließlich führt das Heidentum und die Verbreitung der christlichen Religion als gerechten Kriegsgrund an sowie die nötigenfalls gewaltsame Beseitigung der Hindernisse, die der Christianisierung der Heiden im Wege stehen.

Inferiorität, Immoralität, humanitäre Hilfeleistung und Heidentum sind demnach die zusätzlichen iustae causae für einen Krieg gegen die Indianer. Die beiden ersten Argumente beinhalten eine kulturelle und moralisch-religiöse Diskriminierung, während die beiden letzteren Argumente als Intervention aus humanitären (Verteidigung Unschuldiger) und religiösen Gründen (Rettung vor Verdammnis) zu bestimmen sind.

\subsection{Verhinderung und Bestrafung der Sünden}

Betrachten wir genauer die beiden für die Wahrnehmung der fremden Religion relevanten Argumente, zunächst das der sündhaften Immoralität. Hintergrund dieses Arguments ist die überkommene Lehre von der Sünde als Kriegsgrund, die auf den Kanonisten Sinibaldo Fieschi, den späteren Papst Innozenz IV. $(+1254)$ zurückgeht. Vorkämpfer eines universalen päpstlichen Machtanspruchs, gesteht dieser in seinem Dekretalenkommentar zwar zu, dass es bei den Heiden legitimerweise Eigentum und Herrschaft geben könne, weil diese Güter für jede vernünftige Kreatur bestimmt seien. Daher könne auch der Papst die Heiden nicht einfach aufgrund der infidelitas ihrer Freiheit und ihres friedlichen Besitzes berauben. Doch fügt er zugleich mit Verweis auf das göttliche Gericht über die Sünden Sodoms (Gen 19, 1-29) hinzu, dass ein Heide, der gegen das Naturgesetz verstosse, vom Papst bestraft werden könne, da diesem als Vicarius Christi Gewalt über Gläubige und Ungläubige zukomme. Dasselbe gelte im Fall 
der Idolatrie, da es „natürlich“ sei, nur einen einzigen Gott als Schöpfer zu verehren. Schließlich dürfen die Ungläubigen, so Innozenz, zwar nicht zum Glauben gezwungen werden (non debeant infideles cogi ad fidem), doch könne der Papst den Ungläubigen befehlen, Prediger des Evangeliums in ihrem Territorium zuzulassen; wenn diese aber die Predigt verhinderten, sündigten sie und seien dafür zu bestrafen. Wenn sie aber den Gehorsam verweigerten, seien sie vom weltlichen Arm zu zwingen, und der Papst habe ihnen den Krieg zu erklären. ${ }^{8}$

Dieses theokratische Konzept von der Sünde als Kriegsgrund wurde über mittelalterliche Theologen wie Augustinus von Ancona (Triumphus) weitergegeben und zu Beginn der Neuzeit von Silvestro Prierias (1456-1523), der auch in der causa Martin Luther beschäftigt war, aufgriffen. Politische Bedeutung gewann die Theorie durch Gregorio López, der sie als Mitglied der Junta von Burgos (1512) gutachterlich in die Diskussionen dieses ersten kolonialethischen Beratungsgremiums einbrachte. ${ }^{9}$

Sepúlveda verschärft das Argument, indem er nun auch den Fürsten das doppelte Recht zugesteht, böse Menschen präventiv am Sündigen zu hindern und geschehenes Unrecht (iniuria) zu bestrafen. Verhinderung künftiger Sünden und Bestrafung schon geschehener Sünden, darauf läuft das Argument Sepúlvedas hinaus. Auf den Einwand Leopoldos, dass dieses Recht nur Gott zustehe, erläutert Democrates, dass Gott sein Recht (Röm 13,4) bisweilen durch die Fürsten und die staatliche Obrigkeit ausübe (DS 18).

Mit der mittelalterlichen Kanonistik weiß Sepúlveda, dass der Unglaube (infidelitas), d.h. die andere Religion kein Kriegsgrund sein kann, weil man dafür ebensowenig bestraft werden könne, wie man zum Glauben gezwungen werden dürfe (DS 59). Wohl aber müssten, da Gott bisweilen erlaube, die Sünden der Völker (peccata populorum) zu strafen (DS 26), die schweren Verbrechen der Anthropophagie, der Idolatrie und der Menschenopfer geahndet und zugleich verhindert werden, dass sie künftig begangen werden. Jede einzelne dieser Taten sei allein schon Grund für einen „höchst gerechten Krieg“ (iustissimi belli, DS 39). Sie seien deshalb zu bestrafen, weil sie sich gegen das Naturrecht und die Vernunft richten; und die christlichen Fürsten dürften die Völker unterwerfen, die das Naturrecht verletzen und sie dazu zwingen, die natürliche Ordnung der Dinge einzuhalten (DS $46 \mathrm{f}$ ).

Den entscheidenden Einwand gegen diese Auffassung bringt der Dialogpartner Leopoldo vor, wenn er sagt, dann könne man jedes Land militärisch angreifen, weil das Naturgesetz überall gebrochen werde und überall Sünden

8 Commentaria Innocentii Quarti Pont. Maximi super libros quinque decretalium, Francofurti ad Moenum 1570, f. 430, 430v (In III Decret., tit. 34 de voto, Quod super).

9 Text des Gutachtens in: Bartolomé de Las Casas: Historia de las Indias III, 12 (Obras completas, Bd. 5), Madrid 1994, $1800 \mathrm{f}$. 
begangen würden. Diesem Einwand gegenüber präzisiert Sepúlveda seine Auffassung: Gravierend sei nicht, dass in einem Volk Sünden contra naturam begangen werden, sondern dass die indianischen Völker als ganze, d.h. in ihren Institutionen und Sitten (in publicis moribus et institutis, DS $57 \mathrm{f}$ ) die lex naturalis nicht beachten. Damit versetzt Sepúlveda die altamerikanischen Gemeinwesen in einen Zustand struktureller Sündhaftigkeit.

Die Sünden wider Natur und Vernunft, die Sepúlveda in den indianischen Gemeinwesen individuell gegeben und institutionell verankert sieht, rechtfertigen für ihn den Eroberungskrieg gegen die Indianer. Ziel der Unterwerfung ist die Überführung der Barbarei in Zivilisation, welche wiederum Voraussetzung für die Bekehrung zum Christentum ist. Kriegsziele sind also Zivilisierung und Christianisierung. Der heftigste Gegner dieser Auffassung war Bartolomé de las Casas, der in seiner „Apología“ auch die umfangreichste Widerlegung der von Sepúlveda ins Feld geführten Kriegsgründe verfasst hat. ${ }^{10}$

\section{2 Überwindung des Heidentums}

Für die martialische Überwindung des Heidentums und die Einführung des christlichen Glaubens macht Sepúlveda eine Reihe von Gründen geltend, von denen wir nur die wichtigsten nennen:

(1) Pflichten gegenüber Irrenden. Ein Krieg gegen die Barbaren befolge ein evangelisches Gebot Christi (praeceptum evangelicum) und diene dazu, eine große, im Irrtum befangene Menge von Menschen auf dem schnellsten Weg aus der verderblichen Dunkelheit zum Licht der Wahrheit zu führen. Wenn dies aufgrund des Naturgesetzes und der christlichen Liebe schon für irrende Menschen gelte, um wieviel mehr dann im Fall der Heiden, die zur wahren Religion (veram religionem) zu führen und vor dem Untergang zu retten seien, selbst gegen ihren Willen.

(2) Notwendigkeit präventiver Unterwerfung. Auch wenn niemand zum Glauben gezwungen werden dürfe, sei das Ziel der Verbreitung des christlichen Glaubens zweckmäßigerweise (commode) nicht ohne die Unterwerfung der Barbaren zu erreichen. Diese erleichtere also die folgende Missionierung, und mit demselben Recht könne man unterwerfen und dazu nötigen, das Evangelium zu hören (ad euangelium audiendum compelli). Den Klerikern obliege es, andere zu gerechten Kriegen anzufeuern (inducere alios ad bellandum bella iusta, DS 66).

10 Cf. Bartolomé de las Casas: Apología (Obras completas 9), ed. A. Losada, Madrid 1988, hier 126-356. 
(3) Nötigung zum Glauben. Dem Einwand, dass man zum Glauben nur eingeladen, aber nicht genötigt werden könne, begegnet Democrates mit Verweis auf Paulus, der bei seiner Bekehrung von Christus zum Glauben genötigt worden sei (Apg 9, 3-8). Auch zieht er den klassischen Topos des politischen Augustinismus heran, das lukanische Gleichnis vom Festmahl (Lk 14, 23), bei dem die Zaungäste zum Eintreten genötigt werde (compelle intrare). Augustinus trat zwar grundsätzlich für Toleranz ein und befürwortete Gewaltanwendung nur situativ als ultima ratio, während die Kanonistik des hohen Mittelalters aus diesem zwiespältigen Erbe die Erlaubtheit der Zwangsmittel bei Ketzerbekämpfung und Heidenbekehrung betonte. ${ }^{11}$ Mit Berufung auf diese und andere Autoritäten folgert Sepúlveda, dass die Barbaren nicht nur einzuladen (invitandos), sondern auch zum Guten, d.h. zu Gerechtigkeit und Religion, genötigt werden dürfen (compellendos ad bonum, DS 71), vor allem, wenn dies mit relativ geringem Aufwand zu bewerkstelligen sei. Da jede andere Methode die Missionare der Lebensgefahr aussetze, sei mit Furcht und Schrecken voranzugehen.

(4) Krieg als geringeres Übel. Schließlich gibt Democrates auf Leopoldos Einwand, hier werde versucht, Gutes durch Böses zu erreichen, zwar zu, dass jeder Krieg Schaden und Unrecht verursacht, doch gehe es hier darum, das geringere Übel dem größeren vorzuziehen (levius malum praefertur graviori, DS 77), zumal wenn es um ein doppeltes Gemeinwohl (bonum publicum), nämlich das der Spanier und der Barbaren, gehe. Wenn man Güter und Übel (bona et mala) vergleiche, dann seien erzwungener Herrschaftswechsel und Beraubung gewiss Übel; doch dem stünden jene Güter gegenüber, welche die Barbaren von den Spaniern empfangen hätten: Das nützliche Eisen, das allein schon alles entwendete Gold kompensiere; aber auch das Getreide, die neuen Tierarten (Pferd, Ochse, Esel, Schaf, Ziege) und Pflanzensorten; all das übertreffe an Wert weit alles Gold und Silber, das die Spanier sich angeeignet hätten. Wenn man überdies die Schriftkultur und die christliche Religion hinzurechne, könnten die Barbaren all diese empfangenen Wohltaten kaum je ausgleichen (DS 79).

In Sepúlvedas Argumentationsfolge wird die kulturelle Alterität als Inferiorität und die religiöse Alterität als pekkaminös diskriminiert und pönalisiert. Die kulturelle und religiöse Andersheit haben kein eigenes Recht und können nur kriegerisch bekämpft werden. Diese Position eines ausgeprägten Ethnozentris-

11 Cf. KLaus Schreiner: „Duldsamkeit“ (tolerantia) oder „Schrecken“ (terror). Reaktionsformen auf Abweichungen von der religiösen Norm, untersucht und dargestellt am Beispiel des augustinischen Toleranz- und Gewaltkonzeptes und dessen Rezeption im Mittelalter und in der frühen Neuzeit, in: Dieter Simon (Hg.): Religiöse Devianz. Untersuchungen zu sozialen, rechtlichen und theologischen Reaktionen auf religiöse Abweichung im westlichen und östlichen Mittelalter, Frankfurt 1990, 159-210. 
mus war zwar typisch für die frühe Neuzeit, aber keineswegs unausweichlich, wie andere Zeitgenossen zeigen.

\section{Kommunikation und Mission und bei Francisco de Vitoria}

Der wohl in Burgos geborene Francisco de Vitoria $(1483-1546)^{12}$ studierte als Mitglied des Dominikanerordens in Paris Philosophie und Theologie und lehrte zunächst in Valladolid und dann über zwei Jahrzehnte an der Universität Salamanca. Als „der führende Kopf der aufblühenden spanischen Spätscholastik“13 prägte er mit seinem schulbildenden Denken viele begabte Schüler und reflektierte mit nachhaltigem Erfolg die rechtlichen, ethischen und theologischen Probleme der spanischen Landnahme und der christlichen Mission.

Unmittelbare Anstöße, sich mit diesen Problemen zu befassen, waren für Vitoria Briefe und Berichte über gewaltsame Ereignisse in der Neuen Welt, wie etwa bei der Eroberung des andinen Inkareiches. In einem Brief an Miguel de Arcos vom 8. November 1534 bemerkte Vitoria, dieser höchst ungerechte Krieg richte sich gegen unschuldige Menschen, Nächste und Vasallen des Königs, so dass er nicht sehe, wie diese Tyrannei der Konquistadoren entschuldigt werden könne. $^{14}$

Eine systematische Entfaltung seines diesbezüglichen Denkens finden wir vor allem in einer der 13 erhaltenen, postum veröffentlichten Sondervorlesungen (relectiones), ${ }^{15}$ die sich mit jeweils aktuellen Streitfragen beschäftigten, in Mitschriften der Schüler überliefert wurden und bis heute immer wieder neu aufgelegt werden.

12 Ulrich Horst: Leben und Werke Francisco de Vitorias, in: Francisco de Vitoria, Vorlesungen I (Relectiones), hg. von Ulrich Horst, Hans-Gerd Justenhoven, J. STÜBEN, Stuttgart-Berlin-Köln 1995, 13-99.

13 Wolfanang Reinhard: Geschichte der europäischen Expansion, Bd. 2: Die Neue Welt, Stuttgart 1985, 65.

14 Cf. V. Beltrán de Heredia: Ideas del Maestro Francisco de Vitoria anteriores a las relecciones „De Indis“ acerca de la colonización de América según documentos inéditos, in: Ciencia Tomista 57 (1930) 145-165 (Brief 151-153).

15 Es handelt sich im einzelnen um die Vorlesungen De potestate civili (1528), De homicidio (1530), De matrimonio (1531), De potestate Ecclesiae prior (1532), De potestate Ecclesiae posterior (1533), De potestate papae et concilii (1534), De augmento charitatis (1535), De eo ad quod tenetur veniens ad usum rationis (1535), De simonia (1537), De temperantia (1537), De indis (1539), De iure belli (1539), De magia (1540). Cf. Michael SieVERNICH: Relectiones theologicae, in: Lexikon der theologischen Werke, hg. von M. ECKerT u.a., Stuttgart 2003, 627-628. 


\subsection{Recht auf Andersheit}

Äußerer Anlass für die 1539 gehaltene Sondervorlesung De indis recenter inventis sind die schlechten Nachrichten aus der Neuen Welt. Denn wenn man höre, „daß so viele Menschen getötet, so viele an sich unschuldige Menschen ausgeraubt, so viele Herren von ihren Besitzungen vertrieben und ihres Machtbereichs beraubt wurden, kann man zu Recht fragen, ob diese Vorgänge rechtmäßiger- oder unrechtmäßigerweise geschehen sind." (DI Einleitung Nr. 7; S. 381). ${ }^{16}$

Die Frage nach der Rechtmäßigkeit der spanischen Landnahme ist Teil einer umfassenderen missionstheoretischen Fragestellung, die sich auch aus dem ursprünglich dreiteiligen Plan der Vorlesung ergibt, von dem allerdings nur der erste durchgeführt wurde. Vitoria wollte die Frage beantworten, wie die Spanier sich zu verhalten hätten, damit auch die Indianer das allen Menschen zugedachte Heil erlangen könnten. Dafür waren im einzelnen die Punkte zu klären: (1) aufgrund welchen Rechts (quo iure) die Barbaren unter die Herrschaft der Spanier gekommen sind; (2) welche Befugnisse in zeitlichen und zivilen Angelegenheiten die spanischen Fürsten den Indianern gegenüber haben; (3) welche Befugnisse die Bischöfe oder die Kirche haben, was die geistlichen und die Religion betreffenden Dingen angeht (DI Einleitung; S. 371). Die Vorlesung sollte also Klarheit darüber schaffen, wie man das Ziel der Mission erreichen könne und welches Verhältnis zur politischen und religiösen Ordnung der Indianer dabei zu beachten sei.

Der durchgeführte erste Teil der Vorlesung ist wiederum in drei Teile aufgeteilt, deren erster nach dem Recht der Herrschaft Spaniens in der Neuen Welt fragt. Der zweite Teil erörtert die sieben nach Auffassung Vitorias illegitimen Rechtstitel, während der dritte Teil die sieben legitimen Titel aufführt und begründet. Die Missionsfrage ist bei allen Erörterungen explizit oder implizit mit im Spiel.

Zunächst weist Vitoria argumentativ all jene Diskriminierungen zurück, die Sepúlveda vorgebracht hatte. Dieses Diskriminierungsverbot impliziert das Recht auf Alterität, das unbedingt anzuerkennen ist.

(1) Erstens weist Vitoria, mit Berufung auf das Konzil von Konstanz $(1415)^{17}$, die These zurück, die Bewohner Amerikas würden ihre Herrschafts- und Eigentumsrechte nicht legitim ausüben, weil sie diese durch ihre Verfeh-

16 Francisco de Vitoria: Vorlesungen II (Relectiones). Völkerrecht Politik Kirche, hg. von U. Horst, H.-G. Justenhoven, J. Stüвen (Theologie und Frieden 8), Stuttgart Berlin Köln 1997 (De indis 370-541). Wir zitieren diese neue lateinisch-deutsche Ausgabe im laufenden Text unter Sigel DI sowie interner Zählung und Seitenzählung.

17 Heinrich Denzinger: Kompendium der Glaubensbekenntnisse und kirchlichen Lehrentscheidungen, hrsg. von Peter Hünermann, Freiburg ${ }^{37} 1991$, Nr. 1165. 
lungen gegen das Naturrecht verwirkt hätten, so dass ein christlicher Fürst sie dafür bestrafen könne. Für Vitoria dagegen gilt, dass moralische Verfehlungen oder Sünden keineswegs daran hindern, Herrschaft auszuüben und Eigentum zu haben (non impedit civile dominium, DI I, 3; S. 388).

(2) Zweitens weist er die These zurück, dass aufgrund des Unglaubens (ratione infidelitatis) Herrschafts- oder Eigentumsrechte verlorengingen (DI I, 3; S. 391). Ob „Sünder“ oder „Heide“, aus Gründen moralischen Verhaltens oder religiöser Überzeugungen verliere keiner seine Rechte. Daher folgert Vitoria mit Bezug auf Cajetans (Thomas de Vio) Summenkommentar (II-II q. 66 a. 8), „daß die Barbaren weder wegen sonstiger Todsünden noch wegen der Sünde des Unglaubens daran gehindert werden, echte Herren im öffentlichen wie im privaten Bereich zu sein, und die Güter ihres Landes von den Christen auf dieser Rechtsgrundlage nicht in Besitz genommen werden können" (DI I, 10; S. 399)..$^{18}$

(3) Drittens weist Vitoria die These von der Inferiorität der Indios zurück, aufgrund deren sie als Sklaven „von Natur aus" (servi a natura) deklassiert wurden. Die Tatsache, dass die Indianer eine Ordnung (ordo) ausgebildet haben, d.h. Staaten bilden, über Institutionen wie Ehe und Gesetze verfügen oder die Arbeitsteilung kennen, interpretiert Vitoria als Zeichen des Vernunftgebrauchs (indicium usus rationis); wenn die Indios aber bisweilen dumm erschienen, dann hänge dies nur von einer „schlechten und barbarischen Erziehung" ab (ex mala et barbara educatione), wie sie auch bei der spanischen Landbevölkerung, also „bei uns“ (apud nos) zu finden sei. Überhaupt sei „keiner Sklave von Natur aus“ (nullus est servus a natura) (DI I, 16; S. 405). Kurzum: Die Indianer sind wahre Herren ihrer Länder und dürfen ihrer Herrschaft und ihrer Güter nicht beraubt werden. Sie haben ein Recht auf moralische, religiöse und kulturelle Andersheit und dürfen aus diesen Gründen nicht diskriminiert oder gar bekriegt werden.

Im zweiten Teil der Vorlesung (De Indis II) diskutiert Vitoria sieben Rechtstitel, die für die Rechtfertigung der spanischen Herrschaft in der Neuen Welt vorgebracht wurden, in seinen Augen aber ungeeignet und als unrechtmäßig zu verwerfen sind:

(1) Der Kaiser ist Herr der Welt (imperator est dominus mundi);

(2) der Papst ist auch zeitlicher Herrscher der ganzen Welt (totius orbis monarcha etiam temporalis);

(3) es gibt ein Recht der Entdeckung (iure inventionis);

18 Cf. Antony Pagden: Dispossessing the barbarian: the language of Spanish Thomism and the debate over the property rights of the American indians, in: The languages of political theory in early modern Europe, ed. A. PAGDEN, Cambridge 1987, 79-98. 
(4) die Weigerung, den Glauben an Christus anzunehmen (nolunt recipere fidem Christi);

(5) die Sünden der Barbaren (peccata ipsorum barbarorum);

(6) die freie Annahme der spanischen Herrschaft (per electionem voluntariam) ${ }^{19}$

(7) eine besondere Schenkung Gottes an die spanische Krone (ex speciali dono Dei).

Die beiden ersten Rechtstitel, der cäsarische von der Weltherrschaft des Kaisers und der hierokratische von der Weltherrschaft des Papstes, weist Vitoria ebenso zurück wie bestimmte, dem spanischen König zugeschriebene Herrschaftstitel (dritter, sechster und siebter Titel).

Uns interessieren die beiden Titel, die sich auf Moral und Religion beziehen (vierter und fünfter Titel). Hier kommt es zu einem klaren Bruch mit der Tradition, derzufolge religiöse Andersheit (Idolatrie) als Sünde qualifiziert wurde und entsprechend geahndet werden konnte. Vitoria beendet argumentativ diese Tradition, auf die sich gleichsam ein letztes Mal die Humanisten Juan Ginés de Sepúlveda für die Neue Welt und Alfonso de Valdés für die Alte Welt ${ }^{20}$ berufen. Er verwirft eine moralische und religiöse Diskriminierung der fremden Anderen als „Sünder“ und „Ungläubige“ und bestätigt damit das Recht auf kulturelle und religiöse Andersheit.

Mit dem Recht auf religiöse Alterität ist freilich kein Verzicht auf Mission verbunden, wohl aber eine Bestimmung ihrer Voraussetzungen und Bedingungen. Denn die Adressaten haben ein Recht auf christliche Mission, die freilich bestimmten inneren und äußeren Ansprüchen genügen muss. Für eine authentische christliche Mission formuliert Vitoria eine Reihe von zu beachtenden Grundsätzen:

(1) Unüberwindliche Ignoranz. Zunächst weist er gegen viele Autoritäten nach, dass den Indianern Unkenntnis (ignorantia) und Unglauben (infidelitas) in Bezug auf den christlichen Glauben nicht zur Last gelegt werden dürfen. Vielmehr seien sie aufgrund unüberwindlicher Ignoranz entschuldigt. Überdies gelte das Prinzip, dass sittlich gutes Leben und Handeln nach dem Naturgesetz ethische und religiöse Qualität habe (DI II, 12-14; S. 435441).

(2) Verkündigung ohne Zeichen. In einem weiteren Schritt bestreitet Vitoria die Verpflichtung der Indianer, den christlichen Glauben ohne weiteres,

19 Vitoria bezieht sich hier auf das Requerimiento, ein mit Kriegsandrohung verbundenes Ultimatum, das der Kronjurist Juan López Palacios Rubios verfasst hatte und das ab 1514 vor kriegerischen Handlungen in Amerika zu verlesen war. Cf. Horst Pietschmann, Staat und staatliche Entwicklung am Beginn der spanischen Kolonisation Amerikas, Münster 1980, 65-68.

20 In seinem Dialog zur Rechtfertigung des Sacco di Roma (1527): Alfonso de Valdés: Diálogo de las cosas ocuridas en Roma , ed. José F. Montesinos, Madrid 1928. 
d. h. gleich auf die erste Verkündigung hin (primum nuntium) anzunehmen. Einer solchen bloßen Verkündigung fehle Beweis und Grund zum Glauben (argumentum aut motivum ad credendum). Daher fordert Vitoria für die Verkündigung neben der Glaubwürdigkeit der Verkündiger weitere überzeugende Zeichen, Beweise, Wunder, Motive, Gründe (signa, motiva, miracula, alia probatio, suasio). Eine Verkündigung ohne diese weiteren Zeichen könne die Indianer nicht verpflichten (non tenentur barbari credere). Zur Erläuterung bedient sich Vitoria des Beispiels Islam: Wenn die Sarazenen den Barbaren ihre Religion nur einfach (simpliciter) darlegten, wären letztere nicht zum Glauben verpflichtet; auch wären sie nicht verpflichtet zu entscheiden, welche der beiden nun die "wahrere Religion“ (verior religio) sei. Dafür bedürfe es stärkerer Gründe (probabiliora motiva). Aus all dem folgert Vitoria, dass die Nichtannahme des derart vorgetragenen Glaubens keinerlei Grund für Okkupation oder gar Krieg abgeben könne, denn die Indianer seien unschuldig und hätten keinerlei Unrecht getan (DI II, 15 f; S. $441 \mathrm{f})$.

(3) Kommunikationspflicht. In seiner dritten These vertritt Vitoria eine Pflicht zur Kommunikation. Die Indianer seien gehalten, Missionaren (loquentes de religione) friedlich (pacifice) zuzuhören und sich dann zu beraten (consultare). Falls sie aber nicht hören wollten und für diese Verweigerung keine vernünftigen Gründe beibringen könnten, seien sie nicht von Sünde entschuldigt (DI II, 12).

(4) Glaubensannahme. Darauf aufbauend postuliert Vitoria eine moralische Pflicht zur Annahme des christlichen Glaubens, die allerdings an viele Bedingungen gebunden ist: Der Glaube muss mit glaubwürdigen und einsichtigen Beweisen vorgetragen worden sein (cum argumentis probabilibus et rationalibus); er muss von Menschen vorgetragen worden sein, die ein ehrenhaftes Leben (vita honesta) nach dem Naturgesetz führen; ein solches mit Eifer und Fleiß gelebtes Leben gilt als Bestätigung der vorgetragenen Wahrheit (argumentum ad confirmandam veritatem).(DI II, 18; S. 445)

(5) Mangelnde Zeichen. Vitoria bezweifelt jedoch, dass diese moralische Pflicht zur Annahme des Glaubens faktisch gegeben ist, weil die Bedingungen nicht erfüllt seien, vor allem die nötige Überzeugungskraft (probabili persuasione) fehle. Von Wundern und Zeichen (miracula et signa) höre er ebensowenig wie von Beispielen des Lebens (exempla vitae), wohl aber von Skandalen, Grausamkeiten und vielerlei Ruchlosigkeiten (scandala, saeva facinora, impietates). Obwohl nicht wenige Ordens- und Kirchenleute durch Lebensbeispiel und Predigt viele Mühe aufgewandt hätten, sei die christliche Religion doch nicht derart verkündet worden, dass eine Pflicht zu ihrer Annahme bestünde (DI II, 19; S. 445).

(6) Gewaltverbot. Im letzten Schritt schliesst Vitoria jegliche Gewaltanwendung im Zusammenhang der Glaubensverbreitung aus, weil der Glaube als 
Sache des freien Willens niemals erzwungen werden dürfe. Das gelte auch für den Fall, dass der Glaube zwar glaubhaft verkündet worden ist, die Indianer ihn aber gleichwohl nicht annehmen wollen. Das aber gebe christlichen Fürsten kein Interventionsrecht. Die Hinführung zum christlichen Glauben dürfe nur auf anlockend-gewinnende Weise (blandimentis), nicht aber auf hart-bedrückende Weise (asperitatibus) geschehen. Nach der Praxis der Kirche dürfe die Nichtannahme des Glaubens kein Grund sein, die Ungläubigen mit Krieg zu überziehen, denn der Krieg sei kein Argument für die Wahrheit des christlichen Glaubens und bewege Nichtchristen nicht zur Annahme des Glaubens, sondern nur zur Vortäuschung (ad fingendem se credere) oder bloss äußerlichen Annahme des Glaubens (DI II, 20; S. 447).

Bedingungen jeder missionarischen Aktivität sind nach Vitoria also zum einen die Entfeindung des Verhältnisses zu den Adressaten und zum anderen die Bindung der Verkündigung des christlichen Glaubens an überzeugende Zeichen und glaubwürdige Lebenspraxis.

\subsection{Pflicht zur Kommunikation}

Im dritten Teil der Relectio De indis erörtert Vitoria die sieben geeigneten und rechtmäßigen Titel (tituli legitimi) der spanischen Herrschaft in Amerika. Es sind:

(1) Der Titel der natürlichen Gemeinschaft und Kommunikation (titulus naturalis societatis et communicationis), der das Recht auf Freizügigkeit von Reise und Aufenthalt (ius peregrinandi et degendi) sowie auf Handel (negotiari) einschließt;

(2) die Verbreitung der christlichen Religion (causa religionis Christianae propagandae);

(3) der Schutz der Neuchristen vor Zwangskonversion zur alten Religion;

(4) die päpstliche Einsetzung eines christlichen Fürsten für Neuchristen;

(5) die Verteidigung Unschuldiger vor Tyrannei;

(6) eine wahre und freie Wahl (veram et voluntariam electionem);

(7) die Hilfe für Bundesgenossen und Freunde (causa sociorum et amicorum).

Hier interessiert vor allem der zweite Rechtstitel der Verbreitung der christlichen Religion, der eng mit dem ersten Titel der natürlichen Gemeinschaft und Kommunikation verbunden ist. Ein Recht auf Mission (ius praedicandi) steht damit im Rahmen der freien Kommunikation unter den Völkern, zu der insbesondere das Recht auf Freizügigkeit gehört, weil die Freundschaft mit allen Menschen und die gastliche Aufnahme von Fremden dem Naturrecht ent- 
spreche. Das Recht auf Verbreitung der christlichen Religion entfaltet Vitoria in vier Schritten: ${ }^{21}$

(1) Naturrechtliche Begründung. Zunächst gibt er eine biblische und eine naturrechtliche Begründung für die Mission, d.h. das Recht zu predigen und das Evangelium zu verkünden (ius praedicandi et annuntiandi Evangelium). Biblisch führt er den Missionsbefehl (Mk 16, 15) an, naturrechtlich das Recht, den Willigen die Wahrheit mitzuteilen, soweit diese sich auf Heil (salutem), Glück (felicitatem) und Erziehung (educationem) bezieht. Auch verweist er darauf, dass es aufgrund des natürlichen Rechts auf brüderliche Zurechtweisung (correctio fraterna) und Liebe (dilectio) die Aufgabe gebe, zurechtzuweisen (corrigere) und anzuleiten (dirigere). Schließlich führt er als entscheidenden Grund an, dass es sich bei den Adressaten der Mission um "Nächste“ (proximi) handele, denen gegenüber die Christen die Pflicht hätten, über die göttlichen Dinge aufzuklären (DI III, 8; S. $474 \mathrm{f}$ ). ${ }^{22}$

(2) Päpstliche Sorge. Der nächste Begründungsschritt bezieht sich auf die potestas indirecta des Papstes, der für die Verbreitung des Evangeliums in der ganzen Welt Sorge zu tragen habe (curare promotionem Evangelii) und daher auch einen Fürsten mit dieser Aufgabe betrauen könne. Damit rechtfertigt Vitoria die päpstliche Aufteilung der Interessensphären der iberischen Mächte und das königliche Patronat, ohne indes ausdrücklich auf die einschlägigen päpstlichen Bullen Inter cetera (1493) und Universalis ecclesiae (1508) zu verweisen. ${ }^{23}$ Als entscheidendes Kriterium solcher päpstlicher Akte gilt der Vorteil für die Religion (pro commodo religionis), wodurch Vitoria der religiösen Aufgabenstellung den Vorrang vor allen politischen Rücksichten einräumt. Auf keine Fall darf „das Geschäft des Glaubens und der Konversion der Barbaren" (negotium fidei et conversio barbarorum) gestört werden (DI III, 9; S. 475).

(3) Freie Predigt. Wenn die Indianer den Spaniern gestatten, das Evangelium frei und ungehindert (libere et sine impedimento) zu predigen, dann dürfen weder Krieg noch Okkupation stattfinden (DI III, 11; S. 476).

(4) Intervention bei Unrecht. Wenn aber die freie Verkündigung des Evangeliums behindert wird oder zwar zugelassen, die Bekehrten aber getötet,

21 Cf. Ramón Hernández: Francisco de Vitoria. Vida y pensamiento internacionalista, Madrid 1995, $327 \mathrm{ff}$.

22 Tzvetan Todorov (Die Eroberung Amerikas. Das Problem des Anderen, Frankfurt 1985, 181) unterstellt, dass Vitoria nur „an die Freiheit der Spanier [denkt], den Indianern das Evangelium zu predigen, und nie an die der Indianer das Popol Vuh in Spanien zu verbreiten." Diese Einrede hat allerdings keinen Anhalt an Vitorias Text und Logik der Argumentation.

23 America pontificia primi saeculi evangelizationis 1493-1592. Documenta pontificia ex registris et minutis praesertim in Archivo Secreto Vaticano existentibus, coll., ed. J. Metzler, Città del Vaticano 1991, 71-83, 104-107. 
bestraft oder bedroht werden, dann gestattet Vitoria eine Intervention. Der entscheidende Rechtsgrund für diese Intervention ist jedoch nicht die Andersheit, sondern ein Unrecht (iniuria), das entweder den Spaniern durch Beschneidung ihrer Rechte zugefügt wird oder das Unschuldige durch Einschränkung ihrer religiösen Freiheit erleiden.

Bei allen Interventionsmaßnahmen gelten zudem einschränkende Prinzipien: Krieg ist die ultima ratio, also nur legitim, wenn alle anderen Mittel vorher ausgeschöpft wurden; es gilt das Prinzip der Proportionalität, also der Bewahrung von Maß und Vernunft (servato semper modo et ratione), gegebenenfalls auch Verzicht auf das eigene Recht; jeder Eingriff hat sich mehr am Vorteil für die Indianer (magis ad bonum barbarorum) auszurichten als am eigenen Gewinn; auch dürfe es keinen Verstoß gegen das Evangelium geben. Wenn all dies nicht gewährleistet ist, müsse man von dieser Art der Evangelisierung (ratione evangelizandi) Abstand nehmen und eine andere suchen, zumal zu befürchten sei, dass die Dinge in der Regel weitergetrieben werden als das Recht erlaubt (DI III, 11; S. 477).

Damit schließt Vitoria ein Eingriffsrecht nicht kategorisch aus wie Las Casas, engt es jedoch durch die genannten Bedingungen, den Aufweis der Kontraproduktivität und den Hinweis auf den aristotelischen Satz, dass an sich Erlaubtes durch die Umstände zum Übel werden könne („Nikomachische Ethik" 1106a), derart ein, dass es praktisch nicht mehr anwendbar ist. Eine solche Argumentationsstrategie kann als typisch für Vitoria gelten.

Wenn man die verschiedenen Elemente, die Vitoria zur Sprache bringt, unter missionstheoretischer Rücksicht ordnet, dann ergibt sich folgendes Bild: Auf der Basis naturrechtlich begründeter, für alle Menschen geltender und die Beziehungen der Völkergemeinschaft regelnder Prinzipien entwirft Vitoria die Rahmenbedingungen für die christliche Glaubensverbreitung, für die ihrerseits eine Reihe von unabdingbaren Prinzipien gelten.

$\mathrm{Zu}$ den regulativen Prinzipien zählen die Anerkennung religiöser Alterität und das Verbot der Diskriminierung. Vitoria formuliert diese Prinzipien vor allem deshalb, um die kulturelle, moralische und religiöse Andersheit, wie sie in der Weltgemeinschaft der Völker natürlicherweise gegeben ist, vor unberechtigten Eingriffen zu schützen. Wenn er daher feststellt, dass die Verschiedenheit der Religion (diversitas religionis) kein Kriegsgrund sein darf (De iure belli III, 1; S. 557), so bedeutet dies positiv, dass die religiöse Andersheit naturrechtlich zu respektieren ist.

Aufgrund des Unglaubens (ratione infidelitatis) oder anderer religiöser Überzeugung können für die Andersgläubigen keine Rechte verlorengehen (DI I, 3; S. 391) und für die Christgläubigen keine besonderen Interventionsrechte entstehen. Damit sind Religionskriege prinzipiell ausgeschlossen. 
Das Verbot der Diskriminierung aus religiösen Gründen (Heidentum) oder aus kulturellen Gründen (Barbarei) impliziert die Forderung nach religiöser und kultureller Toleranz. Vitoria bezeichnet die Indianer nach damals üblichem Sprachgebrauch in der Regel als „Barbaren“ (barbari), bisweilen auch als „Heiden“ (pagani). ${ }^{24}$ Doch bei ihm begründen diese Alteritätsmuster ${ }^{25}$ aus naturrechtlichen und theologischen Gründen keine Diskriminierung der fremden Anderen; denn diese werden weder im abschreckenden (Barbar) noch im vorbildhaften Sinn (edle Wilde) für europäische Zwecke instrumentalisiert, sondern in ihrer Andersheit als Gleiche anerkannt: anthropologisch als Menschen (homo homini homo; DI III, 2; S. 465), theologisch als Nächste, (omnem hominem proximum; DI III, 1; S. 465).

Die Anerkennung der kulturellen und religiösen Alterität hat also für Vitoria einen doppelten Grund, aber auch eine Grenze. Sobald bestimmte religiöse oder kulturelle Verhaltensweisen zu Unrecht führen, das anderen Personen, dem Gemeinwesen oder der Weltgemeinschaft der Völker bedrohlich schadet, sind Interventionen aus humanitären Gründen erlaubt. Der einzige Grund (unica et sola causa) für eine solche Intervention ist mithin „erlittenes Unrecht“ (iniuria accepta), das eine Hilfeleistung zugunsten derjenigen erfordert, die unschuldig Unrecht erleiden („De iure belli“ III, 4; S. 551). In diesem Sinn anerkennt Vitoria Tyrannei, Unterdrückung und Unrecht gegen Unschuldige als legitime Interventionsgründe. So können Menschenopfer und Anthropophagie Gründe für eine kriegerische Intervention sein, jedoch nicht aufgrund kultureller Andersheit, wegen religiöser Überzeugungen oder moralischer Verfehlungen, sondern nur deshalb, weil Menschen Unrecht (iniuria) zugefügt wird.

Der frühneuzeitliche Diskurs, ${ }^{26}$ in dem das Verhältnis der Völker, Kulturen und Religionen zueinander zur Debatte stand, legte die Grundlagen zum neuzeitlichen Verständnis der Menschenrechte und des Völkerrechts. Er trug damit zur Überwindung eines ethnozentrischen Dualismus bei, der auf der Normativität des Eigenen und der Diskriminierung des Anderen beruht. In diesem Diskurs kommt vor allem Francisco de Vitoria das Verdienst zu, „die zentralen uni-

24 Zur Entwicklung des Heidenbegriffs cf. Carsten Colpe: Das Siegel der Propheten. Historische Beziehungen zwischen Judentum, Judenchristentum, Heidentum und frühem Islam (ANTZ 3), Berlin 1990.

25 Cf. Wolfgang Reinhard: Der „Andere“ als Teil der europäischen Identität. Vom „Barbaren“ zum „edlen Wilden“. In: Mariano Delgado / Matthias Lutz-BachmanN (Hg.): Herausforderung Europa. Wege zu einer europäischen Identität, München 1995, $132-152$.

26 Cf. José A. Fernández-Santamaria: The State, War and Peace. Spanisch Political Thought in the Renaissance 1516-1559, Cambridge 1977; David Brading: The First America. The Spanish Monarchy, Creole Patriots and the Liberal State 1492-1867, Cambridge 1991, 79-101. 
versalistischen Ideen des christlichen Naturrechts auf internationale und interkulturelle Beziehungen angewandt ${ }^{\text {(27 }}$ und regulative Prinzipien für das Verhältnis der Völker, Kulturen und Religionen zueinander entwickelt zu haben.

(1) Er dämmte die ,gerechten Gründe' für kriegerische Interventionen ein, indem er mit der pekkaminösen Interpretation der Alterität brach und ihre ethnozentrische Diskriminierung delegitimierte.

(2) Er anerkannte die kulturelle und religiöse Alterität, indem er sie auf eine doppelte, eine anthropologische und theologische Grundlage stellte, wonach der Andere „Mensch“ (homo) und „Nächster“ (proximus) und als solcher zu behandeln ist, es sei denn offensichtliches Unrecht (iniuria) gebiete eine humanitäre Intervention.

(3) Er plädierte für symmetrische Beziehungen zwischen den Völkern durch die in der Sozialnatur gründende Kommunikation, von der Freizügigkeit des Reisens (peregrinatio) über die des Handelns (negotiatio) bis zur missionarischen Tätigkeit (praedicatio).

(4) Er forderte eine christliche Mission, die ohne Drohung, Nötigung oder gar Gewaltanwendung auf eine einladend-gewinnende Hinführung zum christlichen Glauben setzt, wobei die Verkündigung mit einer glaubwürdigen Lebensform verbunden sein muss, um das Christentum als die „wahrere Religion“ (verior religio) zu erweisen (DI II, 15; S. 443).

Im Rahmen eines universalen Völkerrechts, das auf Naturrecht und mehrheitlichem Konsens beruht (DI III, 3; S. 467), korrespondiert dem Recht auf kulturelle und religiöse Alterität die Pflicht zur interkulturellen und interreligiösen Kommunikation, die man heute „Dialog" nennt.

27 Vittorio Hösle: Die Dritte Welt als ein philosophisches Problem, in: Ders.: Praktische Philosophie in der modernen Welt, München 1992, 131-165, hier 141. 
